\title{
EL MODELO ARGUMENTATIVO DE TOULMIN COMO ELEMENTO EPISTÉMICO PARA LA PARTICIPACIÓN CIUDADANA: UNA APROXIMACIÓN EN TIEMPOS DE PANDEMIA
}

\begin{abstract}
Toulmin's Argumentative Model as an Epistemic Element for Citizen Participation:
\end{abstract}
An Approach in Times of Pandemic

\begin{abstract}
Juan Carlos García-Cruz, Conacyt / Universidad Autónoma Metropolitana, México correo electrónico: j.carlos.garcia.c@gmail.com
\end{abstract}

Recibido: $18 / 05 / 20$

Aceptado: 22/06/20

Resumen. Este trabajo propone una serie de argumentos filosóficos y epistémicos que incentiven la participación ciudadana fundamentada en las prácticas epistémicas y en el modelo argumentativo planteado por Stephen Toulmin. En la primera parte se dilucida la noción de conocimiento y la manera en que se fundamenta a través de las razones objetivamente suficientes desde las comunidades epistémicas. Posteriormente se desarrolla el concepto de práctica como argumento epistémico para fortalecer la participación ciudadana. Finalmente, apoyándonos en el contexto de la pandemia por SARS-CoV-2 y la enfermedad COVID-19, retomamos elementos del modelo argumentativo de Toulmin para evidenciar que es fundamental una base epistemológica y filosófica para establecer una legítima participación ciudadana para el desarrollo de sociedades basadas en el conocimiento.

Palabras clave: Participación pública, modelo argumentativo, prácticas epistémicas, pandemia, comunidades epistémicas.

\begin{abstract}
This work proposes a series of philosophical and epistemic arguments that encourage citizen participation based on epistemic practices and the argumentative model proposed by Stephen Toulmin. In the first part, the notion of knowledge is elucidated and how it is based on objectively sufficient reasons from the epistemic communities. Later, the concept of practice is developed as an epistemic argument to strengthen citizen participation. Finally, relying on the context of the SARS-CoV-2 pandemic and the COVID-19 disease, we return to elements of Toulmin's argumentative model to demonstrate that an epistemological and philosophical basis is essential to establish legitimate citizen participation for the development of societies. based on knowledge.
\end{abstract}

Keywords: Public participation, argumentative model, epistemic practices, pandemic, epistemic communities. 


\section{Introducción}

En los últimos años, la apropiación social de ciencia, tecnología e innovación (CTI) ha tenido una amplia presencia en artículos dedicados al análisis de políticas públicas de ciencia y tecnología, comunicación de la ciencia, economía, gestión del conocimiento y estudios sociales sobre ciencia y tecnología. No obstante, su abordaje filosófico y epistemológico ha sido poco desarrollado.

Por tanto, ha sido común encontrar matices prescriptivos en el uso del término 'apropiación social del conocimiento', como aquella capaz de fortalecer los procesos de apropiación social de CTI para las intervenciones gubernamentales en el campo de la CTI y en los discursos de comunidades científicas y académicas, en referencia al nuevo contrato social de la ciencia en el siglo XXI y a los propósitos de una mayor pertinencia social del conocimiento. ${ }^{1}$ Por ejemplo, en la convocatoria “Ciencia de Frontera 2019” emitida por el Consejo Nacional de Ciencia y Tecnología (Conacyt) describe como uno de sus objetivos particulares el apoyo a la comunidad científica en México con recursos financieros para que los investigadores en las distintas modalidades desarrollen proyectos que resulten en avances conceptuales del saber científico desde una perspectiva en la que

el conocimiento generado por la investigación, científica, tecnológica y la innovación debe ser utilizado por la sociedad mexicana en su proceso permanente de transformación, desarrollo social y económico. Particularmente dicho conocimiento debe convertirse en motor de desarrollo y en factor dinamizador del cambio social. Por ello, es necesario que las propuestas incluyan, como productos entregables, mecanismos de apropiación social del conocimiento generado por la propuesta. El conocimiento debe socializase para tener un impacto real por lo que la apropiación social del conocimiento debe servir para consolidar a las instituciones que se dediquen a la investigación y a los grupos de investigadores, así como al resto de la población del país. ${ }^{2}$

Como podemos observar, la apropiación social del conocimiento comprende como una situación deseable, aquélla en la que los científicos e investigadores contribuyan no sólo en el avance y generación de conocimiento, sino también para generar e incentivar la cultura científica y la participación ciudadana. En este sentido, la apropiación social de CTI ha dado

\footnotetext{
${ }^{1}$ María Elina Estébañez, "Apropiación social de ciencia y tecnología”, en Universidad y Sociedad. Desafíos de la investigación interdisciplinaria (Buenos Aires: Euduba, 2016), 1.

2 Conacyt, "Convocatoria Ciencia de Frontera 2019". https://www.conacyt.gob.mx/index.php/elconacyt/convocatorias-y-resultados-conacyt/conv-cdf-19/19234-convocatoria-ciencia-de-frontera-19/file.
} 
como resultado la identificación de tres ejes temáticos desarrollados los últimos años: a) la cultura científica y tecnológica; b) la participación ciudadana, y c) el uso de conocimientos.

El primer eje muestra a la cultura científica como una modalidad de la cultura en la que la información representacional o descriptiva, práctica u operacional y valorativa (lo que incluye conocimientos, creencias, valores, prácticas, normas, preferencias, etc.) se refiere a contenidos relacionados con la ciencia. ${ }^{3}$ Esta caracterización incluye tanto la cultura intrínseca de la propia comunidad profesional de los científicos, como a la extrínseca, que se aloja en la sociedad en su conjunto. Es en el ámbito de la cultura científica extrínseca, donde preliminarmente puede definirse a la apropiación como la incidencia de la recepción y asimilación del conocimiento científico y tecnológico sobre las creencias, el lenguaje del sentido común, la racionalidad práctica y sobre la vida cotidiana de las personas. ${ }^{4}$

Un segundo eje se refiere a la participación ciudadana, el cual será central a lo largo de este texto, y que en los últimos años va asumiendo diferentes configuraciones acompañando al profundo cambio observado en los patrones de producción y legitimación del conocimiento. Tradicionalmente, la ciencia y la tecnología eran consideradas actividades propias de los expertos. De aquí se derivaba una visión política sobre estas actividades que sostenía la exclusividad de sus practicantes en la regulación de las actividades: su orientación, evaluación y aplicación pueden $-\mathrm{y}$ deberían- alcanzar interés público. Y, en pos de este objetivo, los mejores calificados para el gobierno de la ciencia y la tecnología son los expertos. ${ }^{5}$

Esta visión sobre la política estaba emparentada directamente con la idea del 'déficit cognitivo' 6 imperante en las concepciones más conservadoras de cultura científica y tecnológica. Actualmente, esta visión está siendo reemplazada por la idea de que las decisiones que involucran a la ciencia y a la tecnología implican no sólo a los expertos sino también a los públicos. De allí a que esta conjunción entre expertos, públicos y política sea

\footnotetext{
${ }^{3}$ Óscar Montañés Perales, "La cultura científica. Un marco conceptual de referencia para la evaluación pública de la ciencia”, en FECYT, Percepción social de la Ciencia y la Tecnología 2010 (Madrid: FECYT, 2011 ), 102.

${ }^{4}$ José Antonio Cerezo y Hurtado Montaña Cámara, "Apropiación social de la ciencia", en FECYT, Cultura científica en Iberoamérica (Madrid: FECYT / RICYT / OEI, 2005), 32.

${ }^{5}$ Estébañez, "Apropiación social”, 6.

${ }^{6}$ Esta concepción presupone que el individuo o individuos no comprenden nociones básicas sobre ciencia y tecnología y, por ende, no es posible su participación en la toma de decisiones.
} 
ubicada en el terreno de la gobernanza, ${ }^{7}$ y que la idea de una comunidad científica de amplia autonomía en la orientación y evaluación de su acción, sea desafiada por la de comunidad ampliada de pares y ciencia posnormal. ${ }^{8}$

Finalmente, un tercer eje se refiere al uso y utilización de los conocimientos generados por la ciencia, tecnología e innovación; específicamente la introducción de representaciones, normas, valores y actitudes propias de las prácticas científicas y tecnológicas - $-\mathrm{y}$, últimamente, de las prácticas tecnocientíficas - en el accionar humano cotidiano o usual (entendiendo por ello a prácticas educativas, agrícolas, deportivas, religiosas, sanitarias, alimenticias, etc.). ${ }^{9}$ Implica procesos de generación y transformación de conocimientos que no están exentos de la intervención de otro tipos de conocimientos como los locales y tradicionales - que forman parte de los acervos culturales de la sociedad. Como resultado, se puede hablar de la hibridación de muchas prácticas sociales que resultan en procesos de innovación. "Si el conocimiento (científico y tecnológico) es adecuadamente apropiado por los diversos grupos sociales que integran la sociedad sobre la cual se aplican las innovaciones, dichas intervenciones podrán expresar más acabadamente la diversidad y riqueza cultural de la sociedad". ${ }^{10}$

Desde esta perspectiva, la participación ciudadana cobra gran relevancia debido a que hace referencia a los mecanismos que impulsan a la democracia participativa que, a diferencia de la democracia representativa, se basan en una mayor incidencia de los ciudadanos en las decisiones del gobierno, sin necesidad de formar parte de la administración pública o de un partido político. Dentro de esta concepción, una mayor participación ciudadana se relaciona con el fortalecimiento de los procesos democratizadores. ${ }^{11}$ Retomando debates de principios del siglo XX entre distintas concepciones filosóficas sobre la relación entre democracia y expertos en Estados Unidos y el modelo de democracia participativa, ${ }^{12}$ se ha caracterizado a la apropiación social de CTI como un

\footnotetext{
${ }^{7}$ Edward Hackett, Olga Amterdamska, Michael Lynch y Judy Wajcman (eds.), The Handbook of Science and Technology Studies (Cambridge, MA: MIT Press, 2008), 429.

${ }^{8}$ Jerome Ravetz y Silvio Funtowitz, Uncertainty and Quality in Science for Policy (Dordrecht: Kluwer, 1990), 14.

9 Javier Echeverría, "Apropiación social de las tecnologías de la información y la comunicación", Revista Internacional de Filosofía, 4, núm. 10 (2008), 174.

${ }^{10}$ León Olivé, "Un tipo de innovación social y cultural: redes para la articulación de usos de la innovación social" en Lucía Merino (ed.), Contexto y usos de la innovación social (Bilbao: Universidad del País Vasco, 2012), 164-165.

${ }^{11}$ Wayne Parsons, Public Policy. An Introduction to the Theory and Practice of Policy Analysis (Cambridge: Edward Elgar Publishing, 1995).

${ }^{12}$ Sabrina McCormick, Mobilizing Science: Movements, Participation and the Remaking of Knowledge 
resultado de los procesos democratizadores aplicados en el campo de la ciencia y la tecnología a través de la extensión de las acciones de participación pública. ${ }^{13}$

Así pues, la participación ciudadana se encuentra intrínsecamente relacionada con la apropiación social de CTI. Por lo tanto, este eje se encuentra más cercano a los aspectos epistémicos y filosóficos que dilucidaremos a lo largo de este trabajo, pues implica la toma de decisiones, el desarrollo de capacidades transformativas de la agencia humana que no se limita al acceso y aplicación de conocimientos, sino a su utilización y transformación para usarlo en la vida cotidiana. No obstante, el punto fundamental es preguntarnos ¿cómo podemos transitar a una participación ciudadana que genere conocimiento e incluso para la resolución de problemas cotidianos que son indispensables para nuestra salud y seguridad?

Por tanto, el presente artículo tiene como objetivo proponer una serie de argumentos filosóficos y epistémicos que incentiven la participación ciudadana fundamentada en las prácticas epistémicas y en el modelo argumentativo planteado por Toulmin. ${ }^{14}$ Así pues, la estructura del artículo se enmarca, en primera instancia en dilucidar qué entendemos por conocimiento y cómo podemos fundamentarlo a través de las razones objetivas suficientes de las comunidades epistémicas. En una segunda aproximación retomamos el concepto de práctica desarrollado en principio por Wittgenstein, Turner, Schatzki y Olivé, ${ }^{15}$ y proponemos argumentos epistémicos que fortalecen la caracterización de la participación ciudadana. Finalmente, proponemos el modelo de racionalidad desarrollado por Toulmin como argumento complementario a la dilucidación de las prácticas epistémicas, apoyándonos en el contexto de la pandemia por SARS-CoV-2 y la enfermedad COVID-19, como parte de la argumentación que se constituye como la base epistemológica y filosófica para establecer una legítima participación ciudadana para el desarrollo de sociedades basadas en el conocimiento.

(Filadelfia, PA: Temple University Press, 2009); Sheila Jasanoff, “Technologies of Humility: Citizen Participation in Governing Science", en Minerva, 41, núm. 3 (2003): 223-244; Eduard Aibar, "La participación del público en las decisiones científico-tecnológicas", en Eduard Aibar, y Miguel Ángel Quintanilla, Ciencia, tecnología y sociedad (Madrid: Trotta, 2012), 303-323.; Brian Wynne, "Misunderstood Misunderstanding: Social Identities and Public Uptake of Science”, Public Understanding of Science, 1, núm. 3 (1992). https://doi.org/10.1088/0963-6625/1/3/004.

${ }^{13}$ Ana Cuevas, "Conocimiento científico, ciudadanía y democracia", Revista Iberoamericana de Ciencia, Tecnología y Sociedad, 4, núm. 10 (2008): 67.

${ }^{14}$ Stephen Toulmin, The Uses of Argument (1958) (Cambridge: Cambridge University Press, 2003).

${ }^{15}$ Ludwing Wittgenstein, Investigaciones filosóficas. Barcelona: Universidad Nacional Autónoma de México / Crítica, 1988; Stephen Turner, The Social Theory of Practices. Chicago: The University of Chicago Press, 1994; Theodore Schatzki, Social Practices. A Wittgensteinian Approach to Human Activity and the Social. Cambridge: Cambridge University Press, 1996; León Olivé, La ciencia y la tecnología en la sociedad del conocimiento. Ética, política y epistemología. México: Fondo de Cultura Económica, 2008. 


\section{en-claves}

\section{La teoría platónica del conocimiento}

La noción sobre el conocimiento viene del problema planteado por Platón en el Teeteto: proporcionar una caracterización adecuada de conocimiento. Por conocimiento se entiende bajo la óptica platónica al conocimiento proposicional, es decir, el que se expresa en la fórmula esquemática:

$\mathbf{S}$ sabe que $\mathbf{P}$

(donde $\mathrm{S}$ es un sujeto epistémico cualquiera y $\mathrm{P}$ una proposición).

La aplicación moderna de la propuesta platónica acerca de lo que es conocimiento consiste en formular tres condiciones necesarias que juntas son suficientes para establecer que alguien sabe algo.

[P] S sabe que P sí y sólo sí:

1) $S$ cree que $P$

2) $P$ es verdadera

3) $S$ tiene razones suficientes para creer que $P$

[o alternativamente; la creencia de $\mathrm{S}$ en $\mathrm{P}$ ] está justificada.

Podemos hacer dos observaciones inicialmente: la definición de la teoría platónica del saber pretende dar pautas de aplicación universal para decidir si un sujeto sabe algo o no. En este sentido, como tantos otros, hay que distinguir netamente entre definición de una noción y el criterio de aplicación de la misma. ${ }^{16}$ Es indudable que la teoría platónica del saber que subyace a $[\mathrm{P}]$, lleva consigo una serie de dificultades. Algunas tienen que ver con la noción de creencia propuesta en la cláusula 1) de [P]. Aquí no nos detendremos en analizar la noción de creencia, por el momento, nos enfocaremos en revisar la cláusula 2), el requisito de verdad. Este requisito parece bloquear cualquier intento de utilizar [P] no como definición, sino como criterio general del saber. En este punto recurrimos a Villoro y el desarrollo de su teoría alternativa del saber con base en la cual formula la siguiente definición.

S sabe que P sí y sólo sí:

1) $S$ cree que $P$

2) $S$ tiene razones objetivamente suficientes para creer que $P$

\footnotetext{
${ }^{16}$ Ulises Moulines, Pluralidad y recursión. Estudios Epistemológicos. (Madrid: Alianza Editorial, 1991), 13. 


\section{enn-claves}

Con respecto a $[\mathrm{P}]$, la cláusula de credibilidad se mantiene. La modificación villoriana consiste en fundir las anteriores cláusulas (2) y (3), en una sola en la que ya no se habla (explícitamente) de verdad, pero en cambio añade su objetividad a la suficiencia de las razones. ¿Es aceptable esta propuesta de cambio para expresar nuestra intuición de lo que es el saber? En principio, estaríamos de acuerdo con Villoro, sin embargo, la raíz del problema es el criterio de verdad y el problema es que no disponemos hasta el momento de un criterio satisfactorio de verdad. ${ }^{17}$

En muchas ocasiones se acusa a Villoro de relativista, pero algunos críticos, como Moulines, ${ }^{18}$ mencionan que el problema es lo que aparece socio-históricamente relativo en su concepto general del saber, es decir, su teoría, ciertamente, no implica una relativización de la verdad, pero sí una relativización del conocimiento. Villoro no es un relativista analítico, pero sí un relativista epistémico, diría Moulines. La noción clave en la que nos detendremos, es la creencia basada en 'razones objetivamente suficientes'. La noción de objetividad es el sustituto de la verdad platónica propuesta en (2) de [P], un sustituto — parece querer decir Villoro- que se acerca lo más posible al requisito sin caer en aquellos extremos inalcanzables del platonismo. Ahora bien, ¿qué entiende Villoro por 'objetividad'? Parece que la objetividad ha de entenderse aquí como equivalente a intersubjetividad. En efecto, vemos lo siguiente:

Una razón es objetivamente para creer si es suficiente (esto es concluyente, completa y coherente) con independencia del juicio de quien lo sustenta. Esa característica podemos reconocerla si la razón es suficiente para cualquier sujeto posible de la comunidad epistémica pertinente. ${ }^{19}$

Evidentemente, Villoro se refiere a las 'razones objetivamente suficientes' para la creencia de un sujeto. Debemos tener claro que existe una diferencia entre la objetividad transubjetiva y la objetividad intersubjetiva: a saber, que mientras que el criterio (inaplicable) para la primera es la concordancia con un hecho objetivo dado independiente de todo sujeto epistémico, en cambio, el criterio para la segunda es el consenso dentro de una comunidad epistémica dada.

\footnotetext{
${ }^{17}$ Por ejemplo, Tarski no proporcionó un criterio general de "verdad" (que es lo que necesitamos para la teoría platónica del saber), sino sólo una definición (restringida) para ciertos lenguajes; b) los supuestos criterios de verdad tradicionalmente propuestos.

${ }^{18}$ Moulines, Pluralidad y recursión, 15.

${ }^{19}$ Luis Villoro, Creer, saber, conocer (México: Siglo XXI, 1982), 112.
} 
Las razones que aduce un sujeto son objetivamente suficientes si son suficientes para cualquier persona a la que le sean accesibles los mismos datos, pueda comprender razones teóricas semejante y acepte el mismo marco conceptual, pero no para otros que no cumplan con esos requisitos; entre aquellas personas se encuentra, naturalmente, el mismo sujeto en cualquier otro momento temporal. Llamemos "sujeto epistémico pertinente" de la creencia de $\mathrm{S}$ en $\mathrm{P}$ a todo sujeto al que le sean accesibles las mismas razones que le son accesibles a $\mathrm{S}$ y no otras, y "comunidad epistémica pertinente" al conjunto de sujetos epistémicos pertinentes para una creencia. ${ }^{20}$

Con lo anterior, es claro que Villoro depende parcialmente de la existencia de comunidades epistémicamente pertinentes. Esta última noción se convierte así en la piedra angular de la teoría villoriana del saber. Por tanto, no bastan con la comprensión de los sujetos epistémicos, sino comprender que son parte fundamental de una comunidad de la que forman parte, estas comunidades son comunidades epistémicas que están socialmente condicionadas. ${ }^{21}$

\section{Comunidades epistémicas}

Villoro plantea y describe a la comunidad epistémica como aquella que se encuentra

[d]eterminada por un nivel de producción específico de su sociedad, que le permite el acceso a ciertos datos mediante ciertos medios técnicos por una cantidad de información acumulada, por un conjunto de teorías e interpretaciones viables, dado el desarrollo alcanzado por el conocimiento de la época, todo ello dentro de un marco conceptual común. ${ }^{22}$

Las comunidades epistémicas están pues condicionadas, tanto en el espacio como en el tiempo. No existe una comunidad intersubjetiva pura de entes racionales posibles; existen intersubjetividades históricamente condicionadas. ${ }^{23}$ Moulines cuestiona la posición de Villoro, ${ }^{24}$ señala un problema fundamental e insuperable con respecto al planteamiento relativista socio-epistémico en la terminología de Villoro. Por tanto, al relativizar la noción de saber a la comunidad epistémica, debemos presuponer un criterio de identidad para las comunidades epistémicas; es decir, necesitamos saber qué son las comunidades epistémicas,

\footnotetext{
${ }^{20}$ Ibid., 147.

${ }^{21}$ Ibid., 150.

${ }^{22}$ Ibid., 147.

${ }^{23}$ Ibid., 149.

${ }^{24}$ Moulines, Pluralidad y recursión.
} 


\section{enn-claves}

identificarlas y distinguirlas de otras. Pero éste es un saber como cualquier otro. Luego tendrá que ser relativo a ¿qué es eso? Aún no sabemos qué es una comunidad epistémica dada, pero necesitamos saberlo para poder saber cualquier cosa en general. Resumiendo, hay que disponer del concepto de comunidad para determinar el conjunto de saber y hay que disponer del concepto de saber para determinar el de comunidad epistémica. Una respuesta provisional proviene de comprender las prácticas epistémicas de cada comunidad. Es decir, entender cómo los sujetos generan los conocimientos y sobre todo como se constituyen en un argumento epistémico. Por ello es indispensable acudir al concepto de práctica para fundamentar las razones objetivamente suficientes.

\section{Sobre el concepto de práctica}

Uno de los críticos de la concepción positivista del conocimiento fue Ludwig Wittgenstein. En sus Investigaciones filosóficas (1988) y luego en Sobre la certeza (1991), Wittgenstein hizo hincapié en la importancia que tiene tomar en consideración las prácticas en distintas áreas de la filosofía y, en particular para elucidar el problema en torno a su relación con el conocimiento. ${ }^{25}$ Estas críticas suscitaron varias preguntas básicas para la teoría del conocimiento, porque si aceptamos la idea de que las prácticas son relevantes para la reflexión epistemológica, entonces cabe preguntarse qué papel desempeñan las prácticas en conexión con la aceptabilidad epistémica; o —específicamente, para este trabajo-, ¿cuál es la importancia de adquirir ciertos conocimientos para una determinada práctica? $\mathrm{Y}$, sobre todo, ¿cómo podemos justificar esos conocimientos como parte del andamiaje epistémico de la participación pública?

En The Social Theory of Practices (1994), Turner indica que una explicación en términos de prácticas requiere que se justifique la inferencia de comportamiento coordinado - manifestada en regularidades observables - a su supuesto origen en presupuestos o prácticas compartidas. ${ }^{26}$ Para Turner, de acuerdo con Olivé, ${ }^{27}$ las prácticas

\footnotetext{
${ }^{25}$ José Antonio Chamizo y Juan Carlos García-Cruz, "Una experiencia en la formación de docentes a partir de la historia y la filosofía de la química", Revista Eureka sobre Enseñanza y Divulgación de las Ciencias, 17, núm. 1 (enero, 2020): 1601-1603. https://doi.org/10.25267/Rev Eureka ensen divulg cienc.2020.v17.i1.1601.

${ }^{26}$ Turner, The Social Theory.
} 


\section{(enn-claves}

sólo podrían entenderse como ocultas y, por lo tanto, resulta imposible dar cuenta de cómo esas prácticas, qua prácticas, se transmiten de individuo a individuo; en lugar de prácticas, debería entonces hablarse de algún tipo de presuposición cognitiva, que es lo que sería compartido, y ésta sería la causa (y punto de acceso al entendimiento) de las manifestaciones compartidas que observamos. Otra crítica de Turner es que el término práctica se utiliza para referirse a muchas cosas: 'tradiciones', 'conocimiento tácito', 'paradigma', 'presuposición', ‘competencias no articulables explícitamente', etcétera. No obstante, Olivé plantea un contraargumento genuino con la idea de cierta clasificación de prácticas, es decir, una práctica depende, siempre, de cierta clasificación de prácticas que da por sentado conocer otras prácticas y, más en general, una serie de presupuestos culturales que permiten distinguir una práctica de otra. ${ }^{28}$ Por ejemplo, en la enseñanza de la química o de la biología las prácticas experimentales son imprescindibles para los alumnos. Son un conjunto de acciones coordinadas por ciertos nexos tales como comprensión de qué hacer y qué decir; conjuntos de reglas, principios, preceptos e instrucciones, o estructuras que incluyen fines, proyectos, tareas, propósitos, creencias, emociones y estados de ánimo. ${ }^{29}$

Esta taxonomía provisional nos lleva a comprender las acciones que se establecen en una práctica experimental. Pero si avanzamos hacia una práctica científica, ésta incluye formas de comprender tipos de acciones; reglas explícitas, principios, preceptos e instrucciones, y estructuras 'teleoafectivas', que contienen jerarquías de fines, tareas, propósitos, proyectos, creencias, emociones y estados de ánimo. Al conjunto de formas de comprensión, reglas y estructura teleoafectiva, Schatzki lo nombra la "organización" de la práctica. ${ }^{30}$

En suma, podemos comprender la práctica como vinculación a sistemas más amplios, ${ }^{31}$ como bien podría entenderse en cuatro grandes contextos de la práctica científica: académica-educativa, investigación, aplicación y evaluación, dentro de cada uno de los cuales y en la interacción entre ellos se producen distintas actividades

${ }^{27}$ Olivé, La ciencia.

${ }^{28}$ Ibid.

${ }^{29}$ Schatzki, Social Practices.

${ }^{30} \mathrm{Ibid}$.

${ }^{31}$ Chamizo y García-Cruz, "Una experiencia”, 1601-1603. 
científicas. ${ }^{32}$ Así pues, la práctica o práctica epistémica, como Olivé la nombra, se constituye como un complejo sistema de actividades (acciones, reacciones, interacciones, hábitos, operaciones, etc.) procedentes de una prolongada tradición cultural: la cultura científica, orientada al logro de conocimientos. ${ }^{33}$ En este sentido, podemos comprender que las prácticas epistémicas están constituidas por grupos humanos cuyos miembros realizan ciertos tipos de acciones buscando fines determinados y, por tanto, además de sujetos (con una subjetividad y emotividad constituida en su entorno cultural), estos seres humanos son agentes, es decir, realizan acciones, proponiéndose alcanzar fines determinados, utilizando medios específicos.

Siguiendo a Olivé, no debemos olvidar que los fines que persiguen los agentes son valorados y las acciones que realizan son evaluadas en función de un conjunto de normas y valores característicos de cada práctica. Así, las prácticas incluyen una estructura axiológica, un conjunto de valores que comparte determinada comunidad, es decir, aquellos valores positivos para la resolución de problemas de determinados sectores. Además, debemos comprender que en todas las sociedades hay prácticas de todo tipo: económicas, técnicas, educativas, políticas, recreativas y religiosas. En las sociedades modernas hay además prácticas tecnológicas y científicas. Pero en todas las sociedades han existido prácticas epistémicas, es decir, aquellas donde se generan conocimiento. Olivé nos menciona que una práctica se entiende como un sistema dinámico con las siguientes características:

a. Un conjunto de agentes con capacidades y con propósitos comunes. Una práctica siempre incluye un colectivo de agentes que coordinadamente interactúan entre sí y con el medio. Por tanto, en las prácticas los agentes siempre se proponen tareas colectivas y coordinadas.

b. Un medio del cual forma parte la práctica, y en donde los agentes interactúan con otros objetos y otros agentes (por ejemplo, el medio donde se realiza una práctica agrícola o pesquera, medicinal o de energía).

c. Un conjunto de objetos (incluyendo otros seres vivos) que forman también parte del medio (semillas, la tierra, especies animales).

d. Un conjunto de acciones (potenciales y realizadas) que están estructuradas. Las acciones involucran intenciones, propósitos, fines, proyectos, tareas, representaciones, creencias, valores, normas, reglas, juicios de valor y emociones. De este conjunto

\footnotetext{
32 Olivé, La ciencia; Echeverría, “Apropiación social”.

${ }^{33}$ Bajo la propuesta conceptual de prácticas epistémicas, Olivé nos menciona que la epistemología puede entenderse como la disciplina que analiza críticamente las 'prácticas epistémicas', es decir, aquellas mediante las cuales se genera, se aplica y se evalúan diferentes formas de conocimiento.
} 
conviene destacar:

d1) Un conjunto de supuestos básicos (principios), normas, reglas, instrucciones y valores, que guían a los agentes al realizar sus acciones y que son necesarios para evaluar sus propias representaciones y acciones, igual que las de otros agentes. Esta es la estructura axiológica de una práctica. ${ }^{34}$

Las prácticas epistémicas, entonces, se desarrollan por grupos humanos y no por individuos aislados. La adecuación de una práctica no es una cosa de todo o nada, sino un asunto gradual que tiene que ver con la medida en que los agentes de la práctica logran los fines que se proponen, y la evaluación de su logro, en función de sus propios valores. Por tanto, es fundamental preguntarnos cómo podemos evaluar esas prácticas. Más específicamente, ¿cómo podríamos validar la apropiación del conocimiento en determinados sujetos que tengan aparentemente comprendidas las prácticas desde la diversidad axiológica de las prácticas epistémicas que se desarrollan en las comunidades epistémicas? Un aporte teórico conceptual que podría incluirse como basamento epistémico-filosófico para incorporar en la participación ciudadana es el modelo de argumentación propuesto por el filósofo inglés Stephen Toulmin para la comunicación y la educación.

\section{La argumentación como vía para la participación pública en tiempos de pandemia}

En el prefacio de la edición de 2003 de The Uses of Argument, Toulmin indica que su intención original, en 1958, era criticar la suposición de muchos filósofos angloamericanos de que cualquier argumento significativo debería expresarse en términos formales. El contraataque de los filósofos académicos fue contundente y terminaron ignorándolo. Sin embargo, pocos años después, fue redescubierto en Estados Unidos por los especialistas en comunicación, convirtiéndose en todo un hito. Desde entonces, el denominado 'modelo de Toulmin' de argumentación ha sido motivo de una gran cantidad de artículos, libros y cursos. ${ }^{35}$

Si bien es cierto, existen diversas formas de argumentar según el campo del saber en el que se construye el argumento (las ciencias, las matemáticas, el derecho, la

\footnotetext{
${ }^{34}$ Olivé, La ciencia; Olivé, "La apropiación social”.

${ }^{35}$ José Antonio Chamizo, "Las aportaciones de Toulmin a la enseñanza de las ciencias", Revista de Enseñanza de las Ciencias: Revista de Investigación y Experiencias Didácticas, 25, núm. 1 (2007): 136.
} 


\section{(en-claves}

comunicación, la economía, la ética). Sin embargo, para Toulmin, hay partes de los argumentos que son generales para todos los campos, y que son las que utilizaremos para la argumentación de un determinado sujeto que aspire genuinamente a lograr una apropiación social del conocimiento. ${ }^{36}$

Supongamos la siguiente situación: un ciudadano promedio de la sociedad global del siglo XXI llega a casa después de una jornada laboral. Enciende el televisor para informarse de algunas noticias relacionadas con su contexto y también con lo que acontece a nivel global. En México, desde el 28 de febrero de 2020, la Secretaría de Salud inició conferencias de prensa a las 19:00, encabezadas principalmente por el Dr. López-Gatell, subsecretario de Salud, con la finalidad de brindar a la población un informe diario sobre el estado del COVID-19. Bajo este contexto, nuestro sujeto X se dispone a escuchar la información sobre la terrible pandemia del SAR-CoV-2 que se originó en la provincia de Wuhan, China, a finales de diciembre de 2019. El subsecretario de Salud comenta lo que ha sucedido a lo largo de estos cuatro meses y explicita que seguimos sin comprender los efectos, las consecuencias $\mathrm{y}$, sobre todo, los tratamientos específicos que genera el virus COVID-19 - como se conoce la enfermedad trasmitida por el virus SAR-CoV-2 - . A lo largo de la conferencia se abordan una serie de medidas preventivas para evitar los contagios masivos y las poblaciones más vulnerables ante la enfermedad COVID-19. En este sentido, hagamos un ejercicio hipotético y supongamos que el subsecretario de Salud menciona que 'tenemos razones para mencionar que el virus SAR-CoV-2 es altamente contagioso. De acuerdo con las evidencias científicas hasta ahora conocidas nos confirman que la población que presenta mayores complicaciones es la mayor a 60 años. Las estadísticas nos muestran una mayor letalidad y mortandad en este grupo; no obstante, en México tenemos otras poblaciones con mucho riesgo como los enfermos crónicos que padecen diabetes, hipertensión, sobrepeso y obesidad además de pacientes que viven otras enfermedades como cáncer o que tiene un sistema inmune muy deprimido o debilitado. Por tanto, pedimos enfáticamente a estas poblaciones que no se expongan y sigan todas las medidas preventivas; es imperativo que se queden en casa para evitar contagios del COVID-19 que puedan llevar a terribles consecuencias'.

En un primer momento, el sujeto $\mathrm{X}$ se pregunta sobre las razones que el especialista tiene sobre la información que acaba de mencionar. Su primera explicación apela a la

\footnotetext{
${ }^{36}$ Toumlin, The Uses of Argument. 
autoridad que tiene el especialista, es decir, es el subsecretario de Salud, especialista en epidemiología, quien tiene conocimiento sobre el tema, aunque no descartemos que cualquier persona puede equivocarse. Más adelante regresaremos a este punto, pero antes continuemos con el ejemplo que puede ponernos en contexto más detalles.

El sujeto $\mathrm{X}$ asiste como todos los días a su trabajo y en un breve descanso para beber un poco de café escucha una conversación del problema que suscita la pandemia. Existe una legítima preocupación entre colegas por la cantidad de noticias respecto al virus SAR-CoV-2 y la enfermedad COVID-19. Unos a otros se preguntan sobre las consecuencias y los problemas de salud que ocasiona la enfermedad COVID-19. En ese momento, el sujeto X emite la siguiente afirmación: "Sabían que el coronavirus es más contagioso con personas mayores de 60 años".

En primera instancia, nos encontramos en una situación similar a la que se enfrentó y experimentó el sujeto $\mathrm{X}$, es decir, él escuchó una serie de afirmaciones en el noticiero, emitidas por un especialista. Por tanto, ambos han emitido una afirmación que requiere un mínimo de argumentación y compromiso que asevera lo dicho. Podemos pensar que dependerá de la seriedad con la que los demás tomen dicha aseveración, ello dependerá de varios factores, por ejemplo, su reputación, su edad, sus títulos académicos, sus ideologías políticas o religiosas. Sin embargo, a pesar de lo anterior, la mejor forma de determinar si una afirmación debe ser considerada prudentemente es revisando la base en la que se sustenta, desafiando sus credenciales y solicitando un argumento que la avale. ${ }^{37}$

Así, en principio, y de acuerdo con el planteamiento platónico, deberían dar las razones y la justificación de dicha afirmación, o como vimos en la primera sección, mínimamente brindar las razones objetivamente suficientes que se dan a favor o en contra de una aseveración. Supongamos que al sujeto $\mathrm{X}$ lo refuta uno de sus compañeros de trabajo, preguntando por qué deberíamos creer en lo dice. Es decir, por qué debemos pensar que el virus afecta solamente a personas mayores de sesenta años. Para Toulmin, es perfectamente normal que la afirmación sea puesta en duda, la persona que la hizo puede y debe apelar a los hechos y presentarlos para demostrar lo que ha dicho. Por tanto, en nuestro ejemplo, el sujeto $\mathrm{X}$ debe demostrar lo que ha afirmado a sus

\footnotetext{
${ }^{37}$ Popper realizó una crítica a esta falacia de autoridad. En la conferencia "Knowledge without authority" Popper menciona que cualquier sujeto está obligado a dar razones sin importar sus credenciales, es más está más obligado. Para más, véase David Miller (Ed.), Popper Selections (Nueva Jersey: Princeton, 1985).
} 


\section{enn-claves}

compañeros de trabajo, en esta misma vertiente también lo tendría que realizar el Subsecretario de Salud mexicano. Pero veamos en qué consiste la propuesta argumentativa de Toulmin.

En primera instancia Toulmin distingue entre la conclusión (C) del argumento y los hechos a los que apelamos como sustento de nuestra conclusión, los datos (D). Ahora bien, la conclusión de un argumento puede ser cuestionada no sólo a partir de los datos que la apoyan sino, por ejemplo, sobre cómo se llegó, con esos datos, a tal conclusión. Así, la tarea ya no es traer a colación más datos sino ciertas reglas, o mejor, afirmaciones hipotéticas que funcionen como puentes entre los datos y la conclusión. Estas afirmaciones suelen ser de la forma: 'dados los datos (D) se puede aceptar que C'. Toulmin llamará a estas reglas o principios: 'garantías' (G). Con lo anterior se tiene el primer esquema que permite analizar los argumentos. Toulmin simboliza con una flecha la relación que hay entre los datos (D) y la conclusión (C) que sustentan. Por otro lado, Toulmin indica la garantía (G) que apoya tal vínculo entre datos y conclusión, escribiéndola debajo de la flecha como podemos verlo en la figura 1.

Figura 1. Modelo de argumentación basado en Toulmin

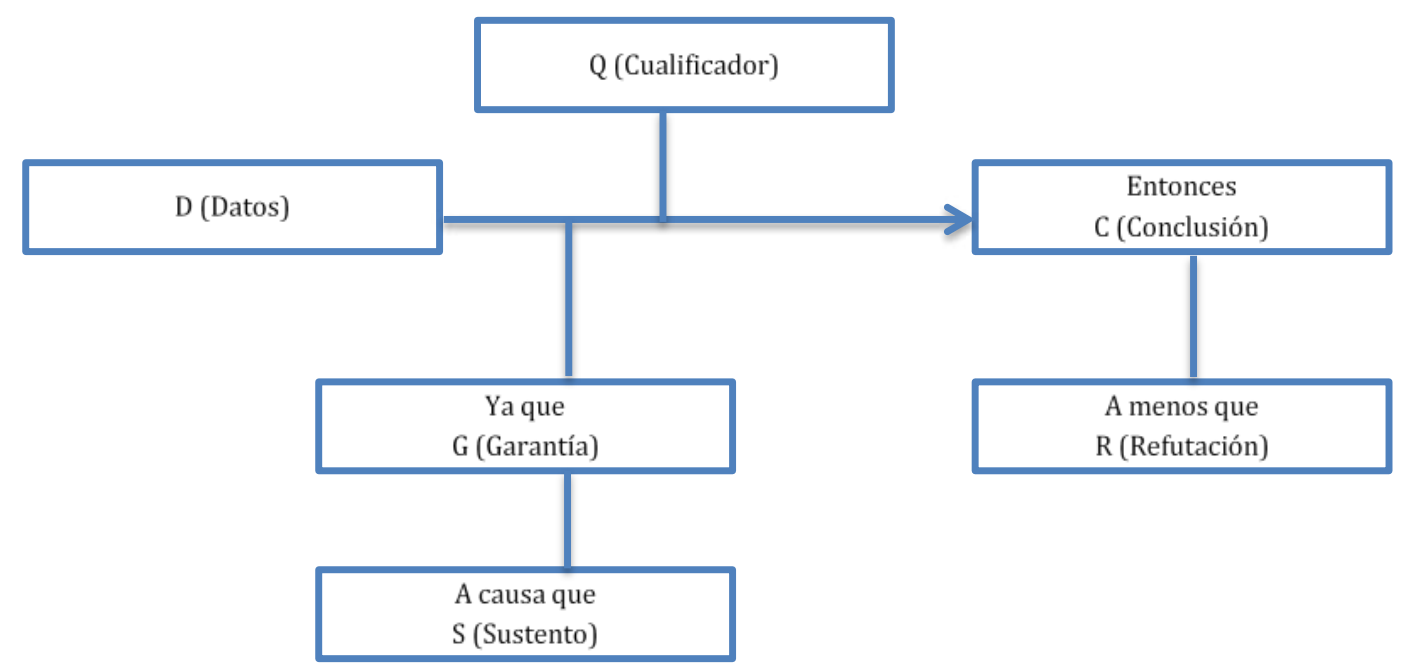

Fuente: Elaboración propia. 


\section{enn-claves}

Revista de Filosofía, Arte, Literatura, Historia

e-ISSN: 2594-1100, Año XIV, Núm. 28, julio-diciembre 2020, 29-51

Así pues, podemos dar cuenta de que el modelo de argumentación de Toulmin, requiere, de manera general, para alcanzar una conclusión el empleo coordinado de la teoría $(\mathrm{G})$ y de la evidencia empírica (D). Hay que hacer notar que en los argumentos se apela explícitamente a los datos para justificar una conclusión. A la garantía se apela implícitamente. Las garantías, además, son generales y certifican la validez de todos los argumentos de un mismo tipo y son establecidas de forma muy distinta a los hechos que usamos como datos para sustentar nuestras conclusiones. Así, y esto es crucial, si en un campo de argumentación algún interlocutor no acepta ninguna de nuestras garantías, entonces será imposible someter los argumentos del campo en cuestión a cualquier tipo de valoración racional. En este caso $(\mathrm{S})$ es el sustento de la evidencia empírica y (R) la refutación. En el modelo de Toulmin el uso de la palabra garantía indica que la validez de la proposición debe ser establecida para garantizar efectivamente la conclusión. Veamos cómo funciona el modelo argumentativo para incentivar la participación ciudadana con la afirmación desarrollada por nuestro sujeto X. A saber: 'Sabían que el coronavirus es más contagioso con personas mayores de 60 años'.

Para ello, es indispensable presentar datos y hechos de la afirmación del sujeto X, podemos determinar que (D) no tiene el grado de fuerza que los datos confieren a la conclusión en virtud de la garantía. Una posible explicación la aporta el concepto de práctica epistémica, es decir, el sujeto X retomó información de un experto, como es el subsecretario de Salud de México. No obstante, este aspecto sólo nos permite comprender en términos más delimitados los contextos y prácticas de los sujetos. Por lo que no sería un impedimento para continuar con una genuina argumentación. Los calificativos modales indican el grado de fuerza que la garantía confiere al paso, y las excepciones y condiciones de refutación indican las circunstancias en que la autoridad general de la garantía tendrá que ser hecha a un lado. En este caso, el punto débil es el (S) de la conclusión y la garantía que avala el paso desde los datos hasta la aseveración final. Por ello también la garantía debe tener sustento (S). Este sustento variará de un campo de la argumentación a otro, de práctica a práctica. Por tanto, aunque la afirmación no cumple a cabalidad con la justificación y las razones en la tríada del conocimiento platónico. No podemos descartar las razones que ha dado el sujeto X. Es decir, él no solamente vio el noticiario, sino que, al observar, comprender y tratar de explicar las 


\section{enn-claves}

evidencias mostradas en la conferencia, logró apropiarse de ciertos conocimientos que incluso le pueden ser útiles en su vida diaria (ver figura 2).

Figura 2. Modelo argumentativo para la participación ciudadana

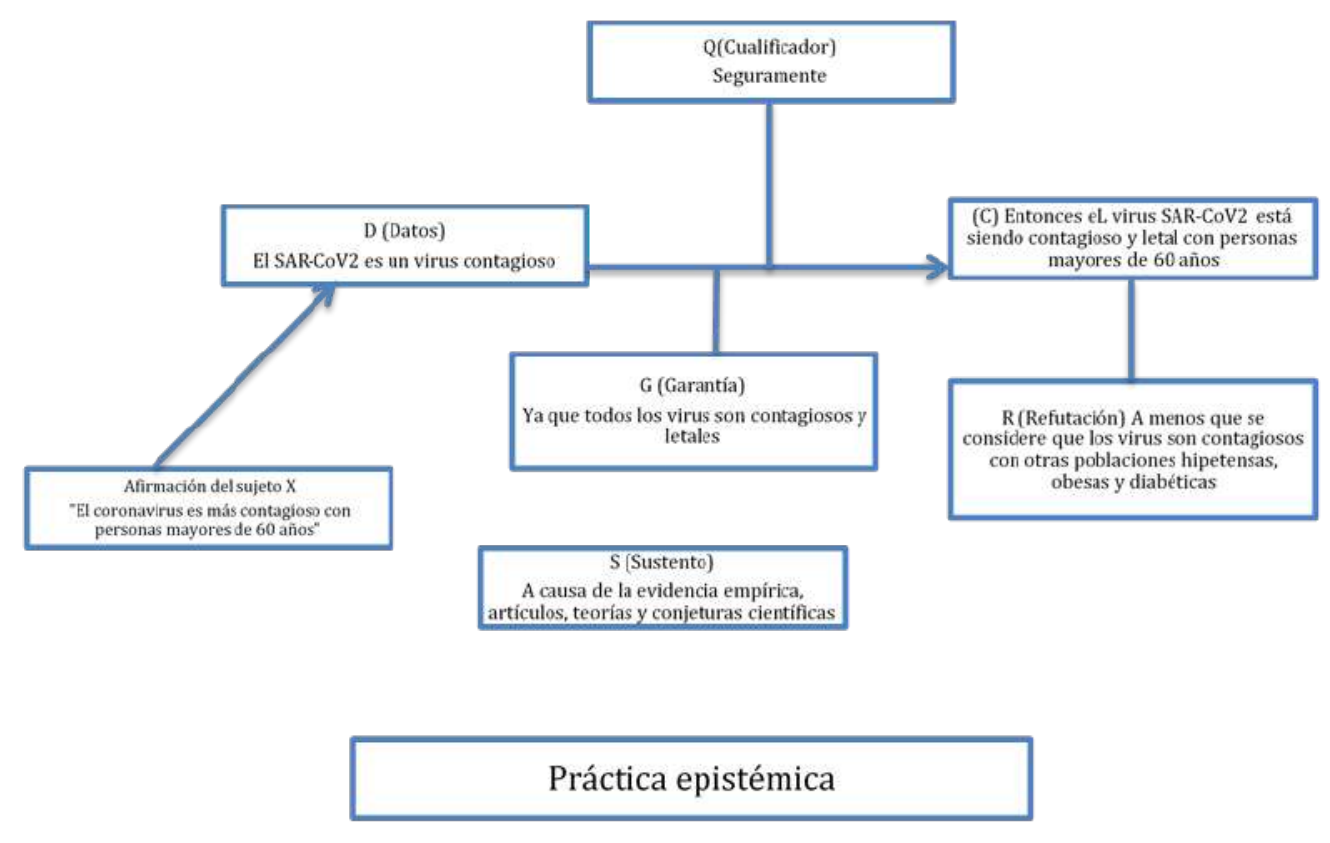

Fuente: Elaboración propia.

Así pues, uno de los aportes de esta propuesta radica en la interacción argumentativa de los sujetos. Pero también en observar en qué grado los sujetos podemos argumentar. Por tanto, un elemento final que puede ayudarnos es tener presente que, bajo la óptica de este modelo de argumentación para incentivar la participación ciudadana, la respuesta siempre va ligada a la pregunta y lo que queremos saber y probar. Así, a partir de tres preguntas diferentes, cualquier sujeto estaría en disposición de argumentar de acuerdo con la secuencia de pensamiento y el desarrollo de sus prácticas: a partir de lo que tenemos, qué se quiere probar y cómo podemos hacerlo, o también qué se quiere probar, a partir de lo que tenemos y cómo podemos hacerlo. ${ }^{38}$ Desde esta óptica, la propuesta no excluye a nadie de una demostración argumentativa, es más, nos permite en cierto modo ver y evaluarnos ante qué tipo de apropiación social de conocimientos estamos trabajando.

\footnotetext{
${ }^{38}$ José Antonio Chamizo, "Las aportaciones de Toulmin a la enseñanza de las ciencias", en Revista Enseñanza de las ciencias: revista de investigación y experiencias didácticas, 25, núm. 1 (2007): 133-146.
} 


\section{Conclusiones}

En los últimos años, la apropiación social de CTI se ha conceptualizado de maneras diferentes, y una conceptualización que nos permite explicar y centrarnos en la discusión, es la elaborada por León Olivé que nos dice que para generar una genuina apropiación social de CTI tenemos que considerar:

1. Apropiación débil: Consiste en la expansión del horizonte de representaciones acerca del mundo por parte del público, que incorpora representaciones provenientes de la ciencia y la tecnología, lo que equivale a la incorporación de representaciones científicas y tecnológicas en la cultura de diferentes miembros de la sociedad.

2. Apropiación fuerte: Va más allá de la incorporación de representaciones provenientes de la ciencia y la tecnología en la cultura de quienes realizan dicha apropiación, para abarcar -lo que es más importante- diversas prácticas sociales (por ejemplo de higiene, sanitarias, productivas o educativas) dentro de la cuales se llevan a cabo acciones propias de esas prácticas que son orientadas por representaciones científicas y tecnológicas del mundo y, en cierta medida, por normas y valores provenientes también de la ciencia y la tecnología. 39

En primera instancia, este tipo de distinción nos permite ir más allá de la apropiación débil en el sentido que los ciudadanos no sólo sean espectadores de la cultura científica, sino también sujetos activos que puedan participar en la generación de mecanismos de participación pública en la toma de decisiones o construcción de agendas de políticas de ciencia, tecnología e innovación. Por tanto, es conveniente preguntarnos ¿cómo desarrollar mecanismos para generar mayor participación ciudadana y transitar a una apropiación fuerte? Olivé nos indica que una vía pertinente es realizarlo a través de tres medios.

i. La comunicación y el periodismo científico de la ciencia y la tecnología, cuya manifestación más débil es la divulgación;

ii. La educación científica y tecnológica en sus diferentes niveles;

iii.y la participación activa en lo que llamaremos redes sociales de innovación. ${ }^{40}$

\footnotetext{
${ }^{39}$ León Olivé, "La apropiación social de la ciencia y la tecnología”, en Tania Pérez Bustos y Marcela LozanoBorda (Eds.), Ciencia, tecnología y democracia: reflexiones en torno a la apropiación social del conocimiento (Medellín: Colciencias / Universidad EAFIT, 2011), 114.

${ }^{40}$ Olivé, "La apropiación social...", 116.
} 
Sin embargo, aunque se han desarrollado trabajos y análisis de caso aún se adolece de los argumentos epistémicos que justifiquen los conocimientos y sobre todo las prácticas para legitimar cada una de las opciones. ${ }^{41}$ Por tanto, a lo largo de este texto se ha presentado una propuesta para lograrlo en la que se toman en cuenta algunas concepciones filosóficas para acercarnos a la apropiación fuerte, vinculada estrechamente a la participación ciudadana; debido a que sin este elemento esencial no podría generarse la apropiación social de conocimientos y por ende, nos veremos imposibilitados para la resolución de problemas específicos.

En los últimos años, se reconoce que la argumentación es importante en el desarrollo de la ciencia ${ }^{42}$ y que el público en general puede y debe participar en los debates en torno a la ciencia si sabe argumentar de manera adecuada. ${ }^{43}$ Podemos observar que el fundamento de las prácticas científicas en la participación ciudadana es indispensable porque permite comprender los distintos contextos y comunidades donde ubicamos la argumentación.

Ante las problemáticas globales a las que se enfrenta la población, es indispensable que los ciudadanos logremos una legítima apropiación social del conocimiento que nos permite incidir para bien en las decisiones de la vida diaria. Parece que después de unos años donde la posverdad era la bandera del siglo XXI hemos regresado o tal vez nos hemos tomado seriamente a las razones que justifican un argumento.

Por lo tanto, podemos identificar algunos niveles de argumentación. El primer nivel estaría justificado por el aprendizaje de algunos conceptos que focalizan el problema. En nuestro ejemplo, el sujeto X estaría en este nivel porque escuchó alguna información, pero la práctica no ayuda mucho porque sólo lo pudo escuchar brevemente y por televisión; tal vez si hubiera asistido o escuchado la conferencia en su versión completa, podría comprender algunos de los conceptos ahí emitidos. El segundo nivel no es exclusivo de la educación, pero quizá ahí es donde el sujeto no sólo se apropia de los conceptos, sino que puede explicarlos sin ningún problema. En gran medida porque está inmerso en esa práctica epistémica.

\footnotetext{
${ }^{41}$ Luz Lazos y León Olivé (Coords.), Hacia un modelo intercultural de sociedad del conocimiento en México. México: Universidad Nacional Autónoma de México, 2014.

42 Anton Lawson, "The Nature and Development Ofhypothetico-predictive Argumentation with Implications for Science Teaching”. International Journal of Science Education, 25, núm. 11 (2003).

43 María Pilar Jiménez-Aleixandre, "Knowledge producers or knowledge consumers? Argumentation and decision making about environmental management”. International Journal of Science Education, 24, núm. 11 (2002): 1171-1190; Jonathan Osborne, Sibel Erduran y Shirley Simon, "Enhancing the quality of argument in school science”. Journal of Research in Science Teaching, 41, núm. 10 (2004): 994-1020.
} 


\section{enn-claves}

Aunque como hemos mencionado, cualquier persona escuchando e investigando por su cuenta puede explicar los conocimientos. Finalmente, un tercer nivel estaría más cercano no sólo a escuchar, comprender y explicar, sino a transformar o modificar ese conocimiento. Estos sujetos son clave porque en un determinado momento puede generar transformaciones de la práctica epistémica, es decir, modificaciones de la misma para beneficio propio o de su comunidad en la resolución de problemas específicos.

Este último nivel es relevante, debido a que es fundamental tener, en principio una mejor comprensión y argumentación para dar cuenta de los fenómenos que nos rodean. Sobre todo, en crisis globales como la pandemia que ahora nos aqueja, esta tarea se vuelve relevante para comprender y explicar los hechos del mundo, contribuyendo a la disminución y distribución de fake news que desinforman a una población. Quizás convendría replantearnos observar con mayor detalle algunos casos para avanzar en esta línea. 


\section{Bibliografía}

Aibar, Eduard. "La participación del público en las decisiones científico-tecnológicas". En Eduard Aibar, y Miguel Ángel Quintanilla, Ciencia, tecnología y sociedad, 303-323. Madrid: Trotta, 2012.

Cerezo, José Antonio, y Montaña Cámara Hurtado. "Apropiación social de la ciencia”. En FECYT, Cultura cientifica en Iberoamérica. Madrid: FECYT / RICYT / OEI, 2005.

Chamizo, José Antonio. "Las aportaciones de Toulmin a la enseñanza de las ciencias". Revista Enseñanza de las Ciencias: Revista de Investigación y Experiencias Didácticas, 25, núm. 1 (2007): 133-146.

Chamizo, José Antonio, y Juan Carlos García-Cruz. "Una experiencia en la formación de docentes a partir de la historia y la filosofía de la química”. Revista Eureka sobre Enseñanza $y$ Divulgación de las Ciencias (2020). https://doi.org/10.25267/Rev Eureka ensen divulg cienc.2020.v17.i1.1601

Conacyt. “Convocatoria Ciencia de Frontera 2019”. https://www.conacyt.gob.mx/index.php/elconacyt/convocatorias-y-resultados-conacyt/conv-cdf-19/19234-convocatoria-cienciade-frontera-19/file

Cuevas, Ana. "Conocimiento científico, ciudadanía y democracia". Revista Iberoamericana de Ciencia, Tecnología y Sociedad, 4, núm. 10 (2008): 67-83.

Echeverría, Javier. "Apropiación social de las tecnologías de la información y la comunicación". Revista Iberoamericana de Ciencia, Tecnología y Sociedad CTS, 4 núm. 10, (2008): 171-182.

Estébañez, María Elina. "Apropiación social de ciencia y tecnología”, en Universidad y Sociedad. Desafios de la investigación interdisciplinaria. Buenos Aires: Eudeba, 2016.

Hackett, Edward J., Olga Amsterdamska, Michael Lynch, y Judy Wajcman (eds.). The Handbook of Science and Technology Studies. Cambridge, MA: MIT Press, 2008.

Jasanoff, Sheila. "Technologies of Humility: Citizen Participation in Governing Science". Minerva, 41, núm. 3 (2003): 223-244.

Jiménez-Aleixandre, María Pilar. "Knowledge producers or knowledge consumers? Argumentation and decision making about environmental management". International Journal of Science Education, 24, núm. 11 (2002): 1171-1190. 
Lawson, Anton. "The Nature and Development Ofhypothetico-predictive Argumentation with Implications for Science Teaching”. International Journal of Science Education, 25, núm. 11 (2003): 1387-1408.

Lazos, Luz, y León Olivé (Coords.). Hacia un modelo intercultural de sociedad del conocimiento en México. México: Universidad Nacional Autónoma de México, 2014.

McCormick, Sabrina. Mobilizing Science: Movements, Participation and the Remaking of Knowledge. Filadelfia, PA: Temple University Press, 2009.

Montañés Perales, Óscar. "La cultura científica. Un marco conceptual de referencia para la evaluación pública de la ciencia”. FECYT, Percepción social de la Ciencia y la Tecnología 2010. Madrid: FECYT, 2011.

Moulines, Ulises. Pluralidad y Recursión. Estudios Epistemológicos. Madrid: Alianza Editorial, 1991.

Olivé, León. La ciencia y la tecnología en la sociedad del conocimiento. Ética, política y epistemología. México: Fondo de Cultura Económica, 2008.

Olivé, León. "Por una auténtica interculturalidad basada en el reconocimiento de la pluralidad epistemológica". En Luis Tapia Mealla (Coord.), Pluralismo epistemológico. Bolivia: CLACSO / CIDES-Universidad Mayor de San Andrés, 2009.

Olivé, León. "La apropiación social de la ciencia y la tecnología”. En Tania Pérez Bustos y Marcela Lozano-Borda (Eds.), Ciencia, tecnología y democracia: reflexiones en torno a la apropiación social del conocimiento. Medellín: Colciencias / Universidad EAFIT, 2011.

Olivé, León. "Un tipo de innovación social y cultural: redes para la articulación de usos de la innovación social”. En Lucía Merino (Ed.), Contexto y usos de la innovación social, 159-170. Bilbao: Universidad del País Vasco, 2012.

Osborne, Jonathan, Sibel Erduran y Shirley Simon. "Enhancing the quality of argument in school science". Journal of Research in Science Teaching, 41, núm. 10, (2004): 994-1020.

Parsons, Wayne. Public Policy. An Introduction to the Theory and Practice of Policy Analysis. Cambridge: Edward Elgar Publishing, 1995.

Ravetz, Jerome, y Funtowitz, Silvio. Uncertainty and Quality in Science for Policy. Dordrecht: Kluwer, 1990. 


\section{(en-claves}

Schatzki, Theodore. Social Practices. A Wittgensteinian Approach to Human Activity and the Social. Cambridge: Cambridge University Press, 1996.

Toulmin, Stephen. The Uses of Argument (1958). Cambridge: Cambridge University Press, 2003.

Turner, Stephen. The Social Theory of Practices. Chicago: The University of Chicago Press, 1994.

Villoro, Luis. Creer, saber, conocer. México: Siglo XXI, 1982.

Wittgenstein, Ludwig. Investigaciones filosóficas. Barcelona: Universidad Nacional Autónoma de México / Crítica, 1988.

Wittgenstein, Ludwig. Sobre la certeza. Traducción de Josep-Lluís Prades y Vincent Raga. Barcelona: Gedisa, 1991.

Wynne, Brian. "Misunderstood Misunderstanding: Social Identities and Public Uptake of Science". Public Understanding of Science, 1, núm. 3 (1992). https://doi.org/10.1088/0963-6625/1/3/004. 